Monsieur

Ueli Müller

Président du Concordat des assureurs-maladie suisses (CAMS)

Römerstrasse 20

4502 Soleure

Berne, le 24 mars 2000

\title{
Communiqué du Concordat des assureurs-maladie suisses sur le TarMed
}

\section{Monsieur le Président,} Mesdames, Messieurs,

C'est avec beaucoup d'étonnement que la Fédération des médecins suisses (FMH) a pris connaissance de votre récent communiqué de presse. De plus, force nous est de constater, dans ce contexte, que jusqu'à aujourd'hui nous n'avons en aucune manière été informés de vos positions ou décisions, ni par téléphone, ni par poste, ni encore par fax ou par e-mail. Il s'agit là d'un procédé quelque peu cavalier, mais nous renonçons, pour notre part, catégoriquement à négocier par médias interposés.

Cela étant, nous vous prions de prendre note des remarques suivantes:

1. Sur le plan opérationnel, les négociations se déroulaient sans problème ces dernières semaines; on pouvait dès lors penser aboutir pour la fin avril à un projet de convention-cadre incluant un concept de neutralité des coûts pour l'introduction des tarifs TarMed et, en particulier, pour un calcul de la valeur initiale du point dans les cantons, respectant cette neutralité des coûts. Mais en donnant aux médias vos "chiffres" et réflexions (que nous renonçons ici à évaluer) en plein milieu de cette phase finale des négociations, vous n'avez fait que recourir à un artifice peu louable gardé en réserve jusqu'ici et sorti pour faire pression sur les négociations.

2. Vous avez, également, systématiquement mal interprété le contenu de la révision 2000 en disant le contraire de ce que vous pensiez: vous savez en effet très bien que cette révision n'est pas une "renégociation" mais qu'elle consiste simplement en un toilettage visant à éliminer les illogismes, lacunes et erreurs rédactionnelles reconnus de part et d'autre. C'est pourquoi d'ailleurs, contre toute attente, les premiers travaux se sont déroulés de manière constructive.

Ce n'est un secret pour personne que la tarification des prestations chirurgicales et interventionnelles est insuffisante et qu'elle demande un réajustement. Nous vous inviterons dès lors à des négociations séparées, ayant pour but une révision dans ce sens.

3. Quant à la lettre restée sans réponse, nous considérons l'affaire comme terminée. Le président de la Fédération s'est expliqué à ce sujet lors de la dernière réunion au sommet et a présenté ses excuses. Dans cette affaire en soi mineure, nous considérons votre attitude comme peu indulgente, pour ne pas dire mesquine, peut-être aussi équivoque, parce qu'elle pousse à se demander si c'est là le seul argument dont vous disposez.

4. Nous tenons, nous aussi, fermement à introduire les nouveaux tarifs jusqu'au 1er janvier 2001 et à créer, du côté de la FMH, toutes les conditions pour l'introduction des structures tarifaires TarMed dans les tarifs cantonaux. A cet égard, nous vous rappelons que la Chambre médicale a donné son aval à la structure tarifaire alpha 2.2 un mois avant vous, que les travaux de conception et d'application revenant à la FMH progressent comme prévu et que près de 2000 médecins ont déjà été formés à l'utilisation du TarMed jusqu'à ce jour. Il devrait être possible de former tout le corps médical d'ici à la fin de l'année. Cela dit, nous ne vous demanderons pas ici où en sont concrètement les travaux préparatoires chez les assureurs.

Comme vous le savez, par son manque de professionnalisme, votre démarche n'a pas manqué d'irriter fortement le corps médical. Nous ne saurions vous cacher qu'un nombre croissant de voix s'élèvent avec véhémence pour remettre en cause ces négociations "partenariales" et réclamer un débat de fond sur la fonction de l'assureur-maladie aujourd'hui. La Fédération devra ainsi convoquer la Chambre médicale à une séance extraordinaire en avril en vue de prendre des décisions sur l'état des négociations avec le CAMS et sur les autres voies praticables.

En prévision de cette prochaine séance de la Chambre médicale, il est indispensable de clarifier la situation au plus haut niveau entre nos organisations; nous vous proposons donc de nous rencontrer, le vendredi 14 avril 2000, le matin ou l'après-midi, dans la région Berne-Soleure. Les modalités exactes de la rencontre seront convenues entre le président du CAMS et celui de la FMH.

Dans l'attente de votre réponse, nous vous prions de croire, Monsieur le Président, Mesdames, Messieurs, à l'assurance de nos sentiments les meilleurs.

Dr H. H. Brunner Président
F.-X. Deschenaux Secrétaire général 